\title{
Fatty acid and metabolomic profiling approaches differentiate heterotrophic and mixotrophic culture conditions in a microalgal food supplement 'Euglena'
}

Min Zeng ${ }^{1}$, Wenlong Hao ${ }^{1,2}$, Yongdong Zou' ${ }^{1}$, Mengliang Shi ${ }^{3}$, Yongguang Jiang ${ }^{1}$, Peng Xiao ${ }^{1}$, Anping Lei ${ }^{1}$, Zhangli $\mathrm{Hu}^{1}$, Weiwen Zhang $^{3}$, Liqing Zhao ${ }^{4^{*}}$ and Jiangxin Wang ${ }^{1,2}$

\begin{abstract}
Background: Microalgae have been recognized as a good food source of natural biologically active ingredients. Among them, the green microalga Euglena is a very promising food and nutritional supplements, providing high value-added poly-unsaturated fatty acids, paramylon and proteins. Different culture conditions could affect the chemical composition and food quality of microalgal cells. However, little information is available for distinguishing the different cellular changes especially the active ingredients including poly-saturated fatty acids and other metabolites under different culture conditions, such as light and dark.

Results: In this study, together with fatty acid profiling, we applied a gas chromatography-mass spectrometry (GC-MS)-based metabolomics to differentiate hetrotrophic and mixotrophic culture conditions.

Conclusions: This study suggests metabolomics can shed light on understanding metabolomic changes under different culture conditions and provides a theoretical basis for industrial applications of microalgae, as food with better high-quality active ingredients.
\end{abstract}

Keywords: Euglena, Metabolomics, Lipids, Fatty acid, Heterotrophic, Mixotrophic

\section{Background}

In recent years, it was found that microalgae are a good source of natural active ingredients [1], and its chemical composition shows a great deal of diversity. Since environmental factors such as temperature, salinity, light, nutrition etc. could affect the chemical composition of microalgal cells, changes in environmental parameters can stimulate or inhibit the biosynthesis of a natural sources of biologically active ingredient [2]. A variety of natural sources of biologically active ingredients from microalgae include carotenoids, phycobilin, fatty acids, polysaccharides, vitamins and sterols [3].

Among them, Euglena is a very promising food and nutritional supplement. This photosynthetic green protozoan

\footnotetext{
* Correspondence: snowyzlj@163.com

${ }^{4}$ Department of Food Science and Engineering, College of Chemistry and Environmental Engineering, Shenzhen University, Shenzhen, Guangdong, China

Full list of author information is available at the end of the article
}

contains no cell wall, so that nutrients inside the cells have a high availability to consumers. It is not only rich in essential poly-unsaturated fatty acids (PUFAs) [4], proteins [5] and antioxidants such as $\beta$-carotene, vitamins $C[6]$ and $E$ $[7,8]$, but also accumulates a large amount of an unique Euglenoid starch-like product paramylon. As a $\beta$-1,3-glucan, paramylon can boost the immune system [9], and sulfated paramylon is also resistant to the effect of HIV [10]. After incorporated into the diet, paramylon can also reduce cholesterol in the blood [11]. Most Euglenoid species have chloroplasts containing chlorophyll a and b and can grow auto-trophic. Some species, such as genus of Euglena (Euglena gracilis), can also bloom in the dark with rich organic water. However, little information is available for distinguishing the different reaction and changes under different culture conditions, such as light and dark.

Compared to transcriptomics and proteomics, metabolomics studies at the cellular level have many advantages 
[12], such as both transcriptomics and proteomics need the complete genome sequences or reference genomes, while metabolomics does not need, and biological pathways are similar, thus metabolomics analyses could be shared across species. With the continuous development of innovative detection techniques and data processing methods, metabolomics research scope has been involved in many fields, such as botany $[13,14]$, food and nutrition sciences [15, 16], toxicology studies [17], clinical diagnosis [18] and more, demonstrating that metabolomics could be a powerful complementation to other "omics" approaches in fully deciphering the metabolic networks.

In this study, we applied a gas chromatography-mass spectrometry (GC-MS) polar metabolite screen as well as fatty acid profiling to analyze cellular responses and changes of E. gracilis under different culture conditions. Significantly different fatty acid compositions were unraveled, and the metabolomics also distinguished the groups under different culture conditions. This study provides a proof-of-concept in how metabolomics can be applied to profile changes and provide a theoretical basis for industrial applications of microalgae as food supplements with high-valued biological active ingredients.

\section{Methods}

\section{Microalgal strains and culture conditions}

Euglena gracilis CCAP 1224/5Z was purchased from CCAP (Culture Collection of Algae and Protozoa) and maintained in our lab at Shenzhen University. This strain is the same as SAG 1224-5/25, UTEX 753, IAM E-6, ATCC 12894 and UTCC 95 in the other culture centers all over the world. The modified heterotrophic acid culture medium [19] was used for E. gracilis. Log phase microalgal cells were inoculated into $250 \mathrm{~mL}$ Erlenmeyer flasks containing $50 \mathrm{~mL}$ fresh medium, at $23 \pm 1{ }^{\circ} \mathrm{C}$ with continuous light of $50 \mu \mathrm{mol} / \mathrm{m}^{2} \mathrm{~s}$ light density as the mixotrophic culture, and aluminum foil was used to wrap the flasks for the dark treatment as the heterotrophic culture. Growth curves and cell numbers were determined by absorbance $750 \mathrm{~nm}$ and the blood cell counting chamber under normal light microscopy, respectively.

\section{Fatty acid composition and quantification}

Total fatty acid extraction and fatty profiling were performed and determined as described by Bligh [20] and Lu [21] with slight modifications, which provided comparative information of the lipid productivities of different cultivation conditions [22], especially used in several microalgae-related researches for lipid extraction [23, 24].

\section{Euglenoid paramylon extraction and quantification}

Paramylon extraction and determination was conducted as previously reported [25], total $5 \mathrm{mg}$ frozen dried cell pellets was used as the input. Followed by acetone enrichment and SDS precipation, paramylon was dried and dissovlved in $0.5 \mathrm{M} \mathrm{NaOH}$. The contents of paramylon were then determined using glucose as the standard.

\section{GC-MS-based metabolomic analysis}

For metabolomic analysis, $\sim 10^{8}$ cells were collected by centrifugation at $8,000 \times \mathrm{g}$ for $10 \mathrm{~min}$ at $4{ }^{\circ} \mathrm{C}$ (Eppendorf 5430R, Hamburg, Germany) from cultures of the $E$. gracilis on day 4, 7, and 9, respectively. The cell pellets were immediately frozen in liquid nitrogen and then stored at $-80{ }^{\circ} \mathrm{C}$ before use. The metabolomic analysis was conducted as described previously $[26,27]$. All chemicals used for metabolome isolation and GC-MS analyses were obtained from Sigma-Aldrich (Taufkirchen, Germany). For metabolomic analysis, cells were collected from samples at day 4, 7, and 9., respectively. The metabolomic analysis protocol included the following. $i$ ) Metabolome extraction: Cells were resuspended in $1 \mathrm{~mL}$ of cold 10:3:1 (v/v/v) methanol/chloroform $/ \mathrm{H}_{2} \mathrm{O}$ solution $(\mathrm{MCW})$, frozen in liquid nitrogen, and thawed five times. Supernatants were collected by centrifugation at $14,000 \mathrm{~g}$ for $3 \mathrm{~min}$ at $4{ }^{\circ} \mathrm{C}$ (Eppendorf 5430R, Hamburg, Germany). To normalize variations across samples, an internal standard (IS) solution $(100 \mu \mathrm{g} / \mathrm{mL}$ U-13Csorbitol, $10 \mu \mathrm{L}$ ) was added to $100 \mu \mathrm{L}$ of supernatant in a $1.5 \mathrm{~mL}$ microtube before it was dried by vacuum centrifugation for $2-3 \mathrm{~h}\left(4{ }^{\circ} \mathrm{C}\right)$; ii) Sample derivatization: the samples were dissolved in $10 \mu \mathrm{L}$ of methoxyamine hydrochloride $\left(40 \mathrm{mg} / \mathrm{mL}\right.$ in pyridine), shaken at $30{ }^{\circ} \mathrm{C}$ for $90 \mathrm{~min}$, added to $90 \mu \mathrm{L}$ of $N$-methyl- $N$-(trimethylsilyl)trifluoroacetamide (MSTFA), and incubated at $37^{\circ} \mathrm{C}$ for $30 \mathrm{~min}$ to trimethylsilylate the polar functional groups. The derivate samples were collected by centrifugation at $14,000 \mathrm{~g}$ for $3 \mathrm{~min}$, and the supernatant was used directly for GC/MS analysis; iii) GC-MS analysis: Analysis was performed on a GC-MS system-GC 7890 coupled to an MSD 5975 (Agilent Technologies, Inc., Santa Clara, CA) equipped with a HP-5MS capillary column $(30 \mathrm{~m} \times 250 \mathrm{~mm}$ id), with $70 \mathrm{eV}$ of electron impact ionization. Two microliters of derivatized sample was injected in splitless mode at $230{ }^{\circ} \mathrm{C}$ injector temperature. The GC was operated at a constant flow of $1 \mathrm{~mL} / \mathrm{min}$ helium. The temperature program started isocratic at $45^{\circ} \mathrm{C}$ for $2 \mathrm{~min}$, followed by temperature ramping of $5^{\circ}$ $\mathrm{C} / \mathrm{min}$ to a final temperature of $280{ }^{\circ} \mathrm{C}$, and then held constant for additional $2 \mathrm{~min}$. The range of mass scan was $m / z$ 38-650; iv) Data processing and statistical analysis: The mass fragmentation spectrum was analyzed using the automated mass spectral deconvolution and identification system (AMDIS) to identify the compounds by matching the data with the Fiehn Library and the mass spectral library of the National Institute of Standards and Technology (NIST). Peak areas of all 
identified metabolites were normalized against the internal standard and the acquired relative abundances for each identified metabolite were used for future data analysis $[26,27]$.

\section{Principal component analysis (PCA) analysis of metabolomics}

All metabolomics profile data was first normalized by the internal control and the cell numbers of the samples, after removing all singlets, i.e. masses detected only in one sample out of the eighteen analyzed, and then subjected to principal component analysis using software SIMCA-P 11.5 .

\section{Statistical analyses}

Significant dissimilarity between groups clustered by PCA were assessed by analysis of molecular variance (ANOVA) based on Euclidean distance using R. The PCA plot obtained for this dataset was characterized by a stress value less than 0.05 , therefore, it could be considered a good representation of the distance matrix as well.

\section{Results and discussion}

\section{Growths under different culture conditions}

The differential growth of E. gracilis under mixo- (HL) and heterotrophic (HD) culture conditions were shown in the Fig. 1. From inoculation to day 6, the fast growing was observed in cultures under both light and dark, with slightly more cells under light condition. It looked like that light had little effect on the growth of E. gracilis during this period. However, from day 7 growth starts to differ from the two conditions: microalgal cells continue to multiply at a lower rate under light, while cells under dark set a downward trend. We speculate that nutrients in the medium were adequate initially to both cells under different culture conditions, thus cells under both conditions were able to vegetative grow and reproduce. Due to the contribution of photosynthesis, so that the

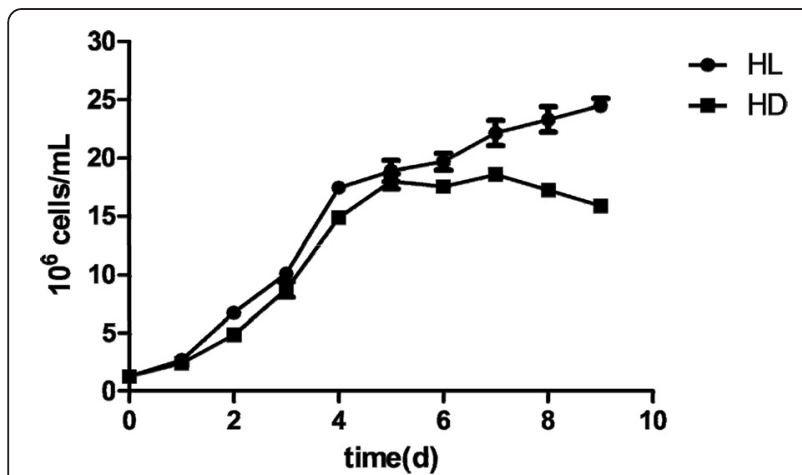

Fig. 1 Growth curves of E. gracilis under mixotrophic (HL) and heterotrophic (HD) conditions growth rate under light is slightly higher than cells under dark. During the period of nutrient depletion, photosynthesis can still support the growth in the light condition, while under dark cell numbers begin to decline due to inadequate nutrition. During culture, cell numbers reached the maximum $2.45 \times 10^{7}$ cells $/ \mathrm{mL}$ and $10.80 \mathrm{~g}$ $\mathrm{dw} / \mathrm{L}$ (dried weight/L) biomass, and it could be possible to obtain higher cell numbers with longer culture time under light. For the cells under dark conditions, cell numbers reached highest at day 7 with $1.86 \times 10^{7}$ cells $/ \mathrm{mL}$ (biomass was $6.61 \mathrm{~g} \mathrm{dw} / \mathrm{L}$ ), with only about 75.9 and $61.2 \%$ of cells under light.

\section{Fatty acid profiling}

This study investigated the fatty acid composition and contents of $E$. gracilis cultured with continuous light and darkness, respectively, at different time points (day $4,7,9$ ). In total, 25 kinds of fatty acids were detected, with carbon chain lengths ranging from 12-22, including 8 saturated fatty acids, and 17 unsaturated fatty acids as shown in Fig. 2 and Table 1.

Accumulation of total fatty acids (TFAs) that were observed increased with the process of culture, under both the light and dark conditions. For instance, the TFA accumulated under light was $108.13 \mu \mathrm{g} / \mathrm{mg} \mathrm{dw}$ at day 4 , while the numbers increased to 127.21 and $136.75 \mu \mathrm{g} / \mathrm{mg} \mathrm{dw}$ at day 7 and 9 respectilvey. Similarly, the TFA contents were 101.30, 137.17 and $161.82 \mu \mathrm{g} / \mathrm{mg} \mathrm{dw}$ at day 4,7 and 9 of dark cultures. In addition, the TFA at the late culture (day 9) in the dark was significantly more than that of the same day cultured under the light.

Interestingly, the fatty acid types under different culture conditions also showed significant difference (Fig. 3), i.e., unsaturated fatty acids (PUFAs) were predominant

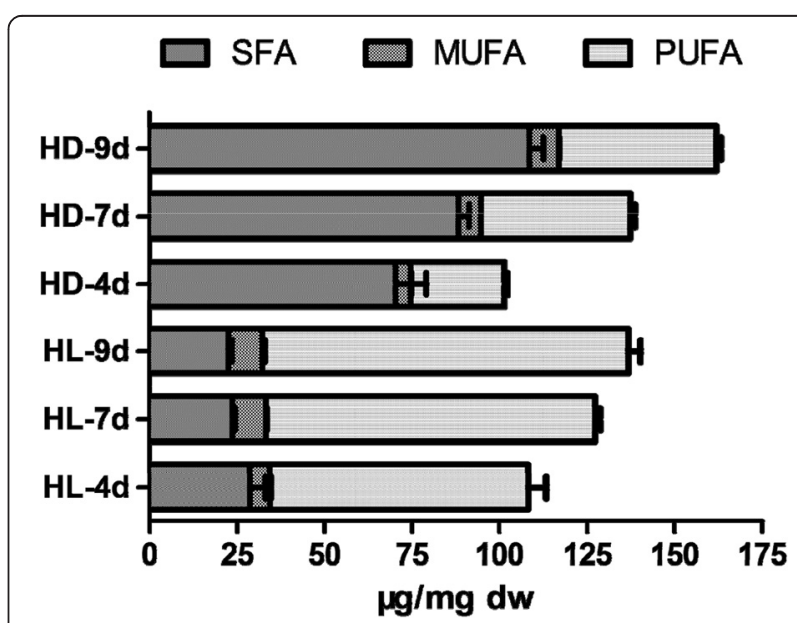

Fig. 2 Detailed fatty acid compostions and contents under different conditions and at different time points. $\mathrm{HL}-4 \mathrm{~d}$, mixotrophic at day 4 . HD-4d, heterotrophic at day 4 
Table 1 Fatty acid compostions ( $\mu \mathrm{g} / \mathrm{mg} \mathrm{dw}$ )

\begin{tabular}{|c|c|c|c|c|c|c|}
\hline & $\mathrm{HL}-4 \mathrm{~d}$ & $\mathrm{HL}-7 \mathrm{~d}$ & $H L-9 d$ & HD-4d & $\mathrm{HD}-7 \mathrm{~d}$ & HD-9d \\
\hline $\mathrm{C} 12: 0$ & $1.54 \pm 0.25^{c}$ & $0.84 \pm 0.05^{d}$ & $0.78 \pm 0.01^{d}$ & $7.29 \pm 0.48^{a}$ & $4.56 \pm 0.03^{b}$ & $4.16 \pm 0.17^{b}$ \\
\hline C13:0 & $1.20 \pm 0.37^{c}$ & $0.71 \pm 0.06^{c}$ & $0.56 \pm 0.04^{c}$ & $7.11 \pm 0.75^{b}$ & $8.10 \pm 0.07^{\mathrm{a}}$ & $7.73 \pm 0.26^{\mathrm{ab}}$ \\
\hline C14:0 & $13.03 \pm 2.93^{d}$ & $8.04 \pm 0.40^{d}$ & $7.49 \pm 0.32^{d}$ & $43.18 \pm 6.72^{c}$ & $54.15 \pm 3.11^{b}$ & $66.11 \pm 2.53^{\mathrm{a}}$ \\
\hline C15:0 & $1.17 \pm 0.21^{d}$ & $1.21 \pm 0.06^{d}$ & $1.06 \pm 0.03^{d}$ & $2.65 \pm 0.19^{c}$ & $5.20 \pm 0.07^{b}$ & $5.62 \pm 0.20^{\mathrm{a}}$ \\
\hline C16:0 & $10.66 \pm 0.63^{c}$ & $11.84 \pm 0.14^{c}$ & $11.66 \pm 0.62^{c}$ & $8.90 \pm 0.52^{d}$ & $14.37 \pm 0.20^{b}$ & $21.65 \pm 0.92^{\mathrm{a}}$ \\
\hline C16:1 & $2.89 \pm 0.23^{c}$ & $5.06 \pm 0.27^{a}$ & $5.45 \pm 0.48^{a}$ & $2.28 \pm 0.20^{d}$ & $3.32 \pm 0.10^{c}$ & $4.58 \pm 0.03^{b}$ \\
\hline C17:0 & $0.30 \pm 0.07^{d}$ & $0.33 \pm 0.02^{d}$ & $0.36 \pm 0.03^{d}$ & $0.56 \pm 0.04^{c}$ & $1.00 \pm 0.04^{b}$ & $1.73 \pm 0.04^{\mathrm{a}}$ \\
\hline C16:2 & $3.79 \pm 0.19^{c}$ & $7.58 \pm 0.07^{b}$ & $10.56 \pm 0.26^{\mathrm{a}}$ & $0.53 \pm 0.03^{e}$ & $0.87 \pm 0.02^{d}$ & $0.78 \pm 0.07^{d}$ \\
\hline C17:1 & $0.63 \pm 0.12^{\mathrm{b}}$ & $1.07 \pm 0.13^{\mathrm{a}}$ & $1.11 \pm 0.17^{a}$ & $0.72 \pm 0.09^{b}$ & $1.06 \pm 0.04^{\mathrm{a}}$ & $1.22 \pm 0.06^{\mathrm{a}}$ \\
\hline C16:3 & $10.96 \pm 0.70^{c}$ & $14.66 \pm 0.59^{b}$ & $16.05 \pm 0.55^{\mathrm{a}}$ & $1.62 \pm 0.07^{e}$ & $2.67 \pm 0.09^{d}$ & $2.05 \pm 0.17^{\text {de }}$ \\
\hline C18:0 & $0.26 \pm 0.06^{\mathrm{cd}}$ & $0.24 \pm 0.03^{c d}$ & $0.22 \pm 0.00^{d}$ & $0.31 \pm 0.03^{c}$ & $0.47 \pm 0.02^{c}$ & $0.85 \pm 0.03^{\mathrm{a}}$ \\
\hline C16:4 & $6.06 \pm 1.11^{b}$ & $7.53 \pm 0.59^{\mathrm{a}}$ & $8.01 \pm 0.88^{\mathrm{a}}$ & $0.12 \pm 0.02^{c}$ & $0.17 \pm 0.01^{c}$ & $0.18 \pm 0.01^{c}$ \\
\hline C18:1 & $2.14 \pm 0.12^{d}$ & $3.39 \pm 0.05^{a}$ & $3.14 \pm 0.17^{b}$ & $1.29 \pm 0.04^{f}$ & $1.91 \pm 0.02^{\mathrm{e}}$ & $2.53 \pm 0.14^{c}$ \\
\hline$C 18: 2 \omega 6$ & $6.00 \pm 0.33^{c}$ & $9.98 \pm 0.12^{b}$ & $12.74 \pm 0.39^{\mathrm{a}}$ & $1.14 \pm 0.06^{\mathrm{e}}$ & $1.71 \pm 0.06^{d}$ & $1.72 \pm 0.05^{d}$ \\
\hline C18:3w3 & $19.40 \pm 1.64^{b}$ & $23.54 \pm 0.63^{\mathrm{a}}$ & $25.03 \pm 1.44^{\mathrm{a}}$ & $1.71 \pm 0.06^{c}$ & $2.51 \pm 0.11^{c}$ & $1.88 \pm 0.01^{c}$ \\
\hline $\mathrm{C} 20: 2 \omega 6$ & $2.50 \pm 0.20^{c}$ & $2.72 \pm 0.23^{c}$ & $2.74 \pm 0.14^{c}$ & $2.66 \pm 0.12^{c}$ & $5.06 \pm 0.19^{b}$ & $5.76 \pm 0.16^{a}$ \\
\hline$C 20: 3 \omega 6$ & $1.47 \pm 0.11^{c}$ & $1.09 \pm 0.04^{d}$ & $0.90 \pm 0.04^{\mathrm{e}}$ & $1.41 \pm 0.05^{c}$ & $1.95 \pm 0.08^{\mathrm{a}}$ & $1.67 \pm 0.05^{b}$ \\
\hline C20:4w6 & $4.02 \pm 0.33^{d}$ & $5.05 \pm 0.25^{c}$ & $5.22 \pm 0.26^{c}$ & $3.86 \pm 0.17^{d}$ & $7.22 \pm 0.25^{b}$ & $8.17 \pm 0.31^{a}$ \\
\hline$c 20: 4 \omega 3$ & $0.74 \pm 0.05^{c}$ & $1.02 \pm 0.03^{b}$ & $1.25 \pm 0.09^{\mathrm{a}}$ & $0.53 \pm 0.02^{e}$ & $0.62 \pm 0.03^{d}$ & $0.64 \pm 0.03^{d}$ \\
\hline $\mathrm{C} 20: 5 \omega 3$ & $5.29 \pm 0.25^{d}$ & $6.07 \pm 0.19^{c}$ & $6.60 \pm 0.28^{\mathrm{ab}}$ & $3.81 \pm 0.10^{e}$ & $6.30 \pm 0.25^{b c}$ & $6.85 \pm 0.14^{a}$ \\
\hline C24:0 & $0.46 \pm 0.07^{b}$ & $0.49 \pm 0.06^{b}$ & $0.42 \pm 0.00^{b}$ & $0.31 \pm 0.04^{c}$ & $0.43 \pm 0.01^{b}$ & $0.67 \pm 0.02^{\mathrm{a}}$ \\
\hline$C 22: 4 \omega 6$ & $0.46 \pm 0.17^{a}$ & $0.26 \pm 0.03^{a}$ & $0.17 \pm 0.01^{b}$ & $0.43 \pm 0.02^{a}$ & $0.47 \pm 0.01^{a}$ & $0.50 \pm 0.02^{b}$ \\
\hline$C 22: 5 \omega 6$ & $6.66 \pm 0.70^{c}$ & $8.07 \pm 0.34^{b}$ & $8.67 \pm 0.22^{\mathrm{ab}}$ & $4.97 \pm 0.15^{d}$ & $7.94 \pm 0.26^{b}$ & $9.12 \pm 0.28^{a}$ \\
\hline $\mathrm{C} 22: 5 \omega 3$ & $0.79 \pm 0.28^{\mathrm{a}}$ & $0.47 \pm 0.02^{\mathrm{bc}}$ & $0.33 \pm 0.01^{c}$ & $0.57 \pm 0.03^{\mathrm{abc}}$ & $0.57 \pm 0.02^{\mathrm{abc}}$ & $0.64 \pm 0.03^{\mathrm{ab}}$ \\
\hline $\mathrm{C} 22: 6 \omega 3$ & $5.73 \pm 0.53^{a}$ & $5.95 \pm 0.21^{\mathrm{a}}$ & $6.23 \pm 0.26^{a}$ & $3.33 \pm 0.05^{c}$ & $4.53 \pm 0.22^{b}$ & $5.01 \pm 0.25^{b}$ \\
\hline Sum & $108.13 \pm 5.87^{c}$ & $127.21 \pm 2.23^{b}$ & $136.75 \pm 4.59^{b}$ & $101.30 \pm 9.46^{c}$ & $137.17 \pm 1.50^{b}$ & $161.82 \pm 5.29^{a}$ \\
\hline
\end{tabular}

$a, b, c, d, e$, and $f$ shows significant difference between each other

under light (68.32-76.41 \% of TFA), followed by saturated fatty acids (SFA) with 16.49-26.46 \%, and monounsaturated saturated fatty acids (MUFA) with 5.23-7.48 \%. Instead, SFAs accounted for 64.36-69.41\% under dark, followed by PUFAs with 26.35-27.79 \%, and MUFAs with $4.24-5.15 \%$ only. As a result, at the same time point, significantly more UFAs (both MUFA and PUFA) were observed in the light cultures than under dark conditions, while for the SFA was the opposite.

In the light culture, contents of PUFAs (Table 1),

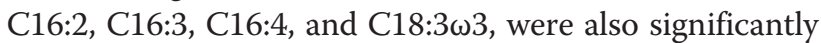
more than those under the dark. In contrast, higher levels of C12:0, C13:0, C14:0 and C16:0 FAs were observed under the dark culture, at all the culture times (day 4, 7 and 9). During the culture process, different FAs showed different trends, for instance, contents of some FAs were increased such as C18: $3 \omega 3$, C16: 3 under light and C14: 0, C16: 0 under dark; others remained unchanged, such as C14: 0, C16 : 0 under light. For the essential fatty acids EPA $(\mathrm{C} 20: 5 \omega 3)$ and

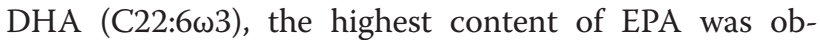
served under dark as $6.85 \mu \mathrm{g} / \mathrm{mg} \mathrm{dw}$, and the content of DHA seemed only connected with light, as they were significantly higher than those under dark, with no significant difference among different culture times at $5.73-6.23 \mu \mathrm{g} / \mathrm{mg} \mathrm{dw}$.

Individually, during the culture process, both $\omega 3$ and $\omega 6$ contents were significantly increased under light and dark groups (Table 2), but $\omega 3 / \omega 6$ ratio was significantly decreased. At the same time point, contents of $\omega 3, \omega 6$ or ratio of $\omega 3 / \omega 6$ under light were significantly higher than the darkness.

In summary, the light does not affect the fatty acids types in E. gracilis, but significantly affects the FA saturation level. In the dark, E. gracilis could accumulate a considerable amount of short-chain FA with the majority of C12-C16:0; In contrast, only under the light conditions, desaturase activity was enhanced to generate a set 
4d

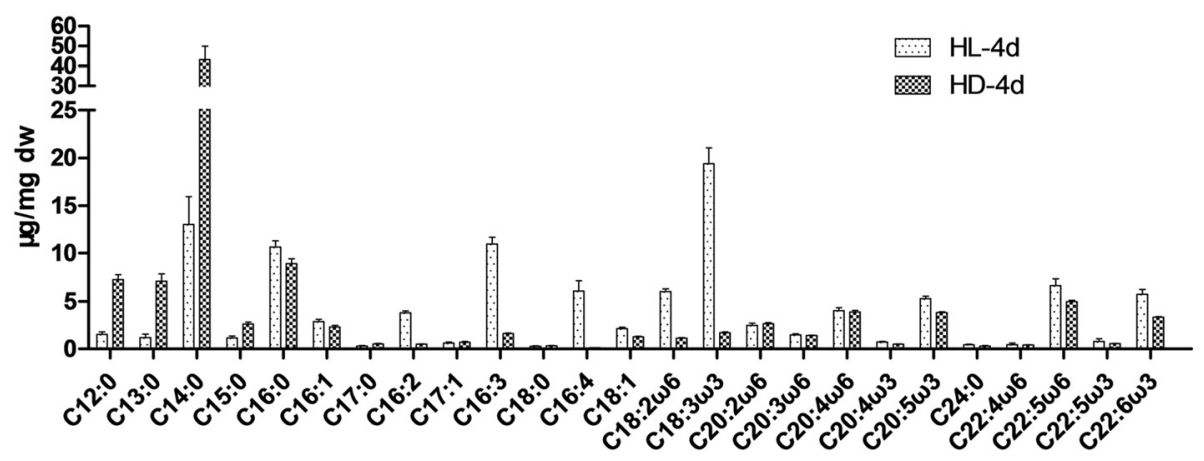

7d

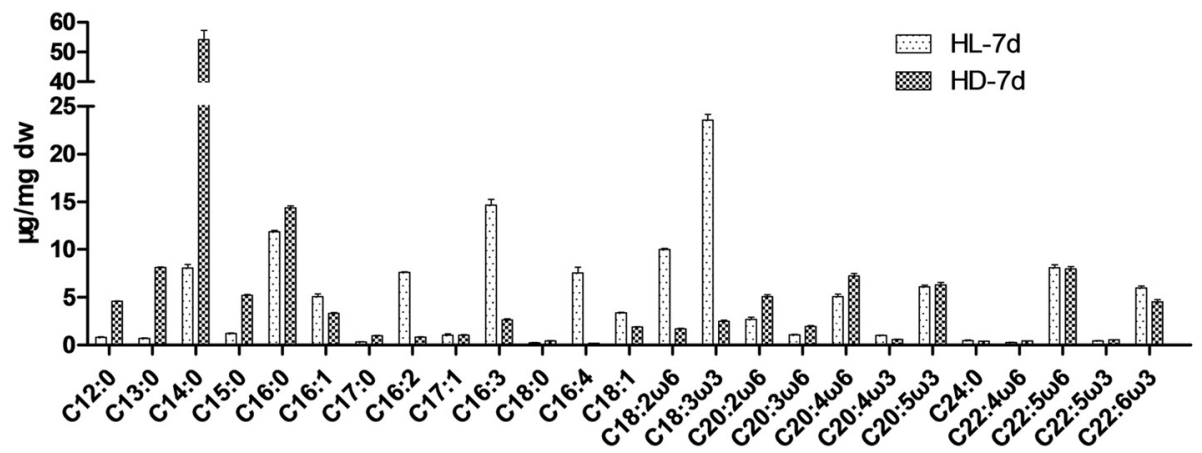

9d

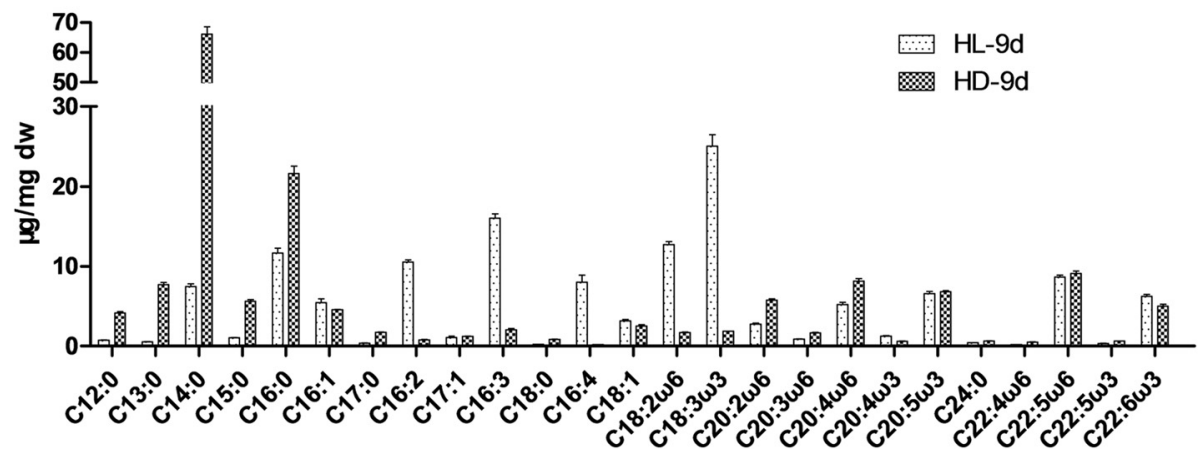

Fig. 3 Total fatty acids, saturated fatty acids (SFA), monounsaturated saturated fatty acids (MUFA) and poly-unsaturated fatty acids (PUFA) under different culture conditions. HL-4d, mixotrophic at day 4; HD-4d, heterotrophic at day 4

of UFAs. The observation in this study agreed with the surveys of Regnault et al. [28] that when carbon source exists Euglenophyta accumulated lots of $\mathrm{C} 14$ and $\mathrm{C} 16$ fatty acids.

\section{Paramylon quantification}

Paramylon, the high-valued product from E. gracilis, was also one of our interests. The highest paramylon contents were observed at day 4 under both conditions compared to the day 7 and 9, showing the decreasing trends in the process of culture (Fig. 4). Expectedly, higher contents of paramylon were observed under dark at days 4 and 7 , while there was no significant difference at day 9 .

It was reported the effect of growth conditions on paramylon contents in wild type and chloroplast-less mutant of E. gracilis [29]. We proposed that paramylon in cells reached a maximum at $24 \mathrm{~h}$ after inoculation, and the contents were then decreased with accelerated growth and reproduction, and probably degraded for the synthesis of other components used in the new cells. Compared to cells under light, cells in the dark 
Table 2 Fatty acids classes and distribution ( $\mu \mathrm{g} / \mathrm{mg} d w)$

\begin{tabular}{|c|c|c|c|c|c|c|}
\hline & $\mathrm{HL}-4 \mathrm{~d}$ & $\mathrm{HL}-7 \mathrm{~d}$ & $\mathrm{HL}-9 \mathrm{~d}$ & HD-4d & $\mathrm{HD}-7 \mathrm{~d}$ & HD-9d \\
\hline$\overline{\text { SFA }}$ & $28.61 \pm 4.31^{d}$ & $23.71 \pm 0.73^{d}$ & $22.55 \pm 1.02^{d}$ & $70.31 \pm 8.71^{c}$ & $88.28 \pm 3.03^{b}$ & $108.53 \pm 4.02^{a}$ \\
\hline MUFA & $5.65 \pm 0.40^{c}$ & $9.51 \pm 0.43^{a}$ & $9.70 \pm 0.81^{a}$ & $4.30 \pm 0.22^{d}$ & $6.29 \pm 0.15^{c}$ & $8.33 \pm 0.19^{b}$ \\
\hline PUFA & $73.87 \pm 5.06^{c}$ & $93.99 \pm 1.52^{b}$ & $104.50 \pm 3.52^{\mathrm{a}}$ & $26.69 \pm 0.87^{e}$ & $42.60 \pm 1.50^{d}$ & $44.96 \pm 1.50^{d}$ \\
\hline SFA $/(M U F A+P U F A)$ & $0.36 \pm 0.07^{d}$ & $0.23 \pm 0.01^{d}$ & $0.20 \pm 0.00^{d}$ & $2.27 \pm 0.23^{\mathrm{a}}$ & $1.81 \pm 0.12^{c}$ & $2.04 \pm 0.06^{b}$ \\
\hline$\omega 3$ & $31.95 \pm 2.26^{c}$ & $37.05 \pm 0.61^{b}$ & $39.44 \pm 1.85^{\mathrm{a}}$ & $9.95 \pm 0.26^{\mathrm{e}}$ & $14.53 \pm 0.59^{d}$ & $15.02 \pm 0.45^{d}$ \\
\hline$\omega 6$ & $21.11 \pm 1.40^{d}$ & $27.17 \pm 0.90^{b}$ & $30.44 \pm 0.96^{\mathrm{a}}$ & $14.48 \pm 0.54^{\mathrm{e}}$ & $24.35 \pm 0.82^{c}$ & $26.93 \pm 0.83^{b}$ \\
\hline$\omega 3 / \omega 6$ & $1.51 \pm 0.03^{\mathrm{a}}$ & $1.36 \pm 0.04^{b}$ & $1.30 \pm 0.05^{c}$ & $0.69 \pm 0.01^{d}$ & $0.60 \pm 0.00^{e}$ & $0.56 \pm 0.00^{\mathrm{e}}$ \\
\hline
\end{tabular}

$a, b, c, d$, and e shows significant difference between each other

accumulated much more paramylon at the beginning but they were consumed very quickly to the similar levels as under light.

\section{Metabolomic analysis}

Regarding the polar metabolite profiling, for metabolomics, we total obtained in total 18 sets of data, 9 for each culture condition and triplicates for each data point. Under this experiment two different culture conditions, a total of 86 metabolites were obtained. Overall, the three biological replicates of each sample was relatively distributed close to each other, and different samples under the same culture condition were separated from one another, indicating the reliability and reproducibility of metabolomics approaches in this study. Under different culture conditions, HL and HD groups could be significantly separated based on the metabolites (Fig. 5a). HD groups from different time points could be distinguished, but with a smaller difference as those between HL and HD. Surprisingly, groups under light at day 7 and 9 were assorted very closely, indicating few metablomic changes under these time points.

GC-MS based metabolomics analysis can achieve a good coverage of polar metabolites, such as amino acids and organic acids, and allow analysis of a wide range of chemical metabolite classes in a single run [30]. In the previous study, we developed an

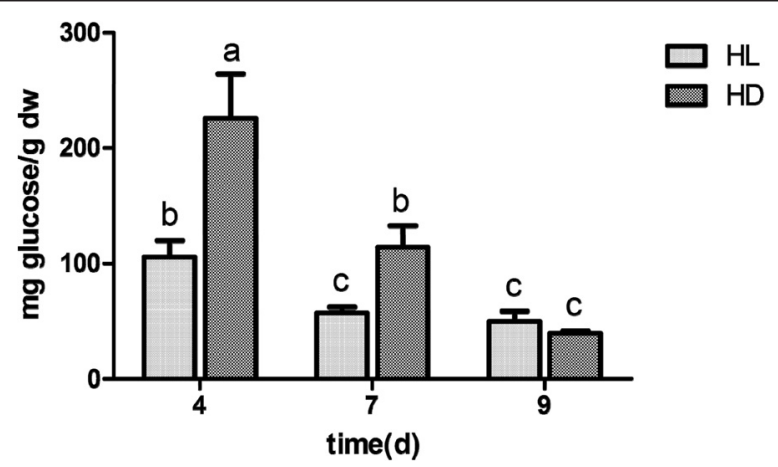

Fig. 4 Paramylon contents under different culture conditions. a, b, and $\mathrm{c}$ show significant difference between each other optimized protocol characterizing the time-series metabolic responses for metabolite isolation and MS analysis, and achieved identification of more than 65 , 60 and 111 chemically classified metabolites from Escherichia coli [26], cyanobacterium Synechocystis sp. PCC 6803 [31], and heterotrophic dinoflagellate microalga Crypthecodinium cohnii [32], respectively. In this study, we followed the similar protocol with minor modification by collecting the cells under light and dark culture at day 4, 7 and 9. A good separation of intracellular metabolites was achieved on the GC column and further MS analysis allowed the chemical classification of a total of 86 metabolites from E. gracilis, including various fatty acids, amino acids, sugars and organic acids. Metabolites detected in E. gracilis are much more than those in E. coli and Synechocystis, probably due to E. gracilis is a eukaryote.

PCA score plots were first applied to evaluate the similarities and differences between a total of 86 metabolomic profiles (Fig. 5b). In general, the score plots of the GC-MS metabolomic profiles revealed overall good reproducibility between biological replicates and good separation between different sample clusters. Combined with previous growth data, this result suggested that members of the light cultivated groups had significantly different metabolisms. Hence GC-MS could be used to reflect cells metabolism changes caused by different culture conditions.

PCA score plots revealed that 86 detected metabolites were plotted in the intermediate position, indicating that no dramatic metabolite changes occurred in the culture throughout the process. However, the distributions of some compounds were relatively significant, which may be associated with different culture conditions. These metabolites were dioctyl phthalate and putrescine. Interestingly, there is no report about the impact of dioctyl phthalate on cellular metabolism or photosynthesis. The emergence of this metabolite is most likely an artifact from extraction/derivatization processes. Diamines and polyamines such as putrescine and spermidine are specific regulators of cellular and metabolic processes which can stimulate active transport of metabolites, and affect 


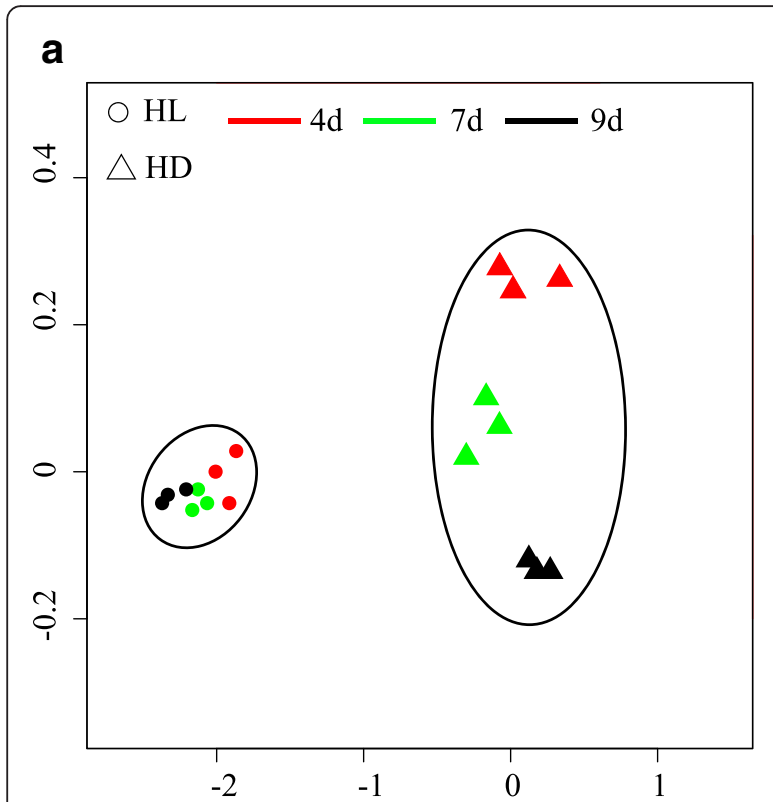

\section{b}

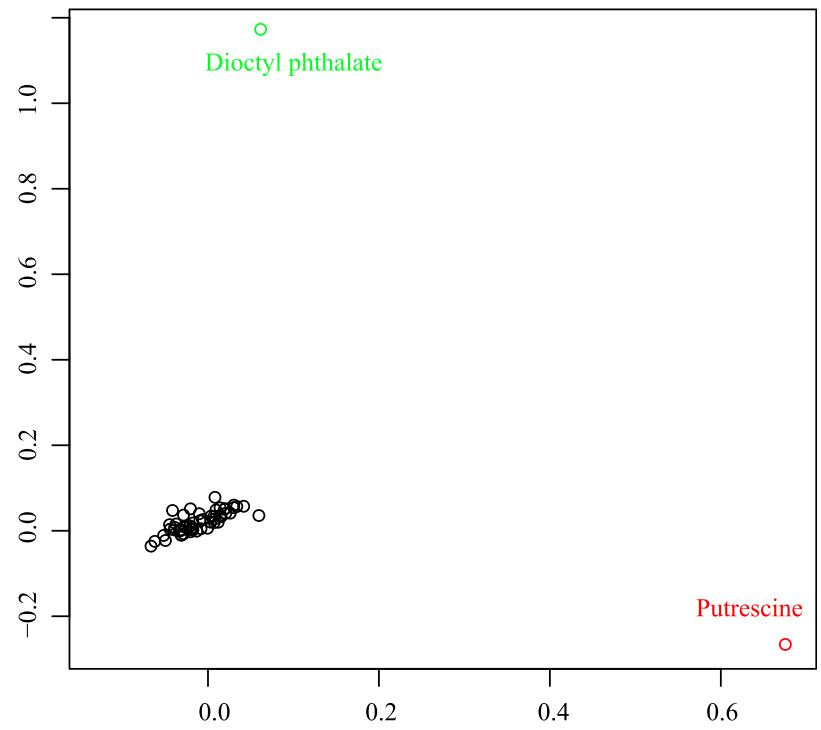

Fig. 5 Metabolomic analysis of cells under different culture conditions. PCA plots of group clusters (a) and all metabolites (b)

the functioning of enzymes and ion pumps in the cellular membranes. They also stimulate the photosynthetic process in green microalgae [33]. Thus, putrescine could be the potential target bio-stimulator for E. gracilis growth.

\section{Conclusions}

In summary, different culture conditions in E. gracilis produced significant different profiles of fatty acids and metabolites. Compared to cells under light, cells under dark accumulated much more paramylon at the beginning of cultivation. It was also shown in this study that the light does not affect the fatty acids types in E. gracilis, but significantly affects the FA saturation level. In the dark, E. gracilis could accumulate a considerable amount of short-chain FA, but only under the light conditions, desaturase activity was enhanced to generate a set of UFAs. The GC-MS could reflect cell metabolism changes caused by different culture conditions and the potential target bio-stimulator, putrescine, for E. gracilis growth was also detected via metabolomic analysis in this study.

\section{Abbreviations}

dw/L, dried weight/L; FAs, fatty acids; HD, heterotrophic; $H L$, mixotrophic; MUFA, monounsaturated saturated fatty acids; PCA, principal components analysis; PUFAs, poly-unsaturated fatty acids; SFA, saturated fatty acids; TFA, total fatty acid

\section{Acknowledgement}

We thank Drs. Chen Huirong, Wang Chaogang, and Li Hui in our lab at Shenzhen University for their assistance for the culture and technical support.

\section{Funding}

This work was supported by the Natural Science Foundation of SZU (\#827-000081), National Natural Science Foundation of China (\#31470431), Guangdong Natural Science Foundation for Major cultivation project (\#2014A030308017), Shenzhen Grant Plan for Science \& Technology (JCYJ20120613112512654 and JCYJ20140418182819134), the National High-tech R\&D Program (863 Program, \#2012AA02A707) funded by the Ministry of Science and Technology of China, and Nanshan District key lab for biopolymers and safety evaluation (\#KC2014ZDZJ0001A), used for the design of the study, data collection, data analysis, interpretation of data, and in writing the manuscript, respectively.

\section{Availability of data and material}

The data and material are available as supplementary data in BMC website.

\section{Authors' contributions}

$Z L, Z M, H W, S M$ and WJ participated in the design of experiments, collected the data and drafted the manuscript. $Z Y, Z M, J Y$ and $H Z$ participated in data collection. $\mathrm{LA}, \mathrm{ZL}, \mathrm{HZ}$ and WJ participated in the design of experiments and helped write the manuscript. $L A, X P, Z L$, $Z W$ and WJ coordinated the research and helped to finalize the manuscript. All authors read and approved the final manuscript.

\section{Competing interest}

The authors declare that they have no competing interests.

\section{Consent for publication}

Not applicable.

Ethics approval and consent to participate

Not applicable.

\section{Author details}

'Shenzhen Key Laboratory of Marine Bioresource \& Eco-environmental Science, Shenzhen Engineering Laboratory for Marine Algal Biotechnology, College of Life Science, Shenzhen University, Shenzhen 518060, People's Republic of China. ${ }^{2}$ Nanshan District key lab for biopolymers and safety evaluation, Shenzhen University, Shenzhen 518060, People's Republic of China. ${ }^{3}$ Laboratory of Synthetic Microbiology, School of Chemical Engineering and Technology, Tianjin University, Tianjin 300072, People's Republic of China. ${ }^{4}$ Department of Food Science and Engineering, College of 
Chemistry and Environmental Engineering, Shenzhen University, Shenzhen, Guangdong, China.

Received: 25 January 2016 Accepted: 24 May 2016

Published online: 02 June 2016

\section{References}

1. Plaza M, Cifuentes A, Ibáñez E. In the search of new functional food ingredients from algae. Trends Food Sci Technol. 2008;19(1):31-9.

2. Marinho-Soriano E, Fonseca P, Carneiro M, Moreira W. Seasonal variation in the chemical composition of two tropical seaweeds. Bioresour Technol. 2006;97(18):2402-6.

3. Gouveia L, Raymundo A, Batista AP, Sousa I, Empis J. Chlorella vulgaris and Haematococcus pluvialis biomass as colouring and antioxidant in food emulsions. Eur Food Res Technol. 2006:222(3-4):362-7.

4. Barsanti L, Bastianini A, Passarelli V, Tredici MR, Gualtieri P. Fatty acid content in wild type and WZSL mutant of Euglena gracilis. J Appl Phycol. 2000; 12(3-5):515-20

5. Kitaoka S, Hosotani K. Studies on Culture Conditions for Determination of Nutritive-Value of Euglena-Gracilis Protein and General and Amino-Acid Compositions of Cells. J Agric Chem Soc Jpn. 1977;51(8):477-82.

6. Takeyama H, Kanamaru A, Yoshino Y, Kakuta H, Kawamura Y, Matsunaga T. Production of antioxidant vitamins, $\beta$-carotene, vitamin $C$, and vitamin $E$, by two-step culture of Euglena gracilis Z. Biotechnol Bioeng. 1997;53(2):185-90.

7. Kusmic C, Barsacchi R, Barsanti L, Gualtieri P, Passarelli V. Euglena gracilis as source of the antioxidant vitamin E. Effects of culture conditions in the wild strain and in the natural mutant WZSL. J Appl Phycol. 1998;10(6):555-9.

8. Ogbonna JC, Tomiyamal S, Tanaka H. Heterotrophic cultivation of Euglena gracilis Z for efficient production of a-tocopherol. J Appl Phycol. 1998;10(1): 67-74.

9. Kondo Y, Kato A, Hojo H, Nozoe S, Takeuchi M, Ochi K. Cytokine-Related Immunopotentiating Activities of Paramylon, a. BETA.-(1. RAR. 3)-D-Glucan from Euglena gracilis. J Pharmacobiodyn. 1992;15(11):617-21.

10. Koizumi N, Sakagami H, Utsumi A, Fujinaga S, Takeda M, Asano K, Sugawara I, Ichikawa S, Kondo H, Mori S. Anti-HIV (human immunodeficiency virus) activity of sulfated paramylon. Antiviral Res. 1993;21(1):1-14.

11. Wang L, Behr SR, Newman RK, Newman CW. Comparative cholesterollowering effects of barley $\beta$-glucan and barley oil in golden Syrian hamsters. Nutr Res. 1997;17(1):77-88.

12. Taylor J, King RD, Altmann T, Fiehn O. Application of metabolomics to plant genotype discrimination using statistics and machine learning. Bioinformatics. 2002;18 suppl 2:S241-8.

13. Dan M, Gao X, Xie G, Liu Z, Zhao A, Jia W. Metabolomic analysis in plant research. J Chin Tradit Med. 2008;32:2337-41.

14. Hall RD. Plant metabolomics: from holistic hope, to hype, to hot topic New Phytol. 2006;169(3):453-68.

15. Zhong K. Metabolomics in food safety and nutrition studies. Foreign Mecine. 2009: 93-97.

16. Wishart DS. Metabolomics: applications to food science and nutrition research. Trends Food Sci Technol. 2008;19(9):482-93.

17. Bai J, Cheng Y. Metabolomics in new drug toxicity mechanism studies. J Chin Med. 2006;40:1601-4.

18. Li H, Jiang Y, He F. Metabolomics in clinic research. Genet. 2008;30:389-99.

19. Wolken JJ. Euglena; an experimental organism for biochemical and biophysical studies. 1961

20. Bligh EG, Dyer WJ. A rapid method of total lipid extraction and purification. Can J Biochem Physiol. 1959;37(8):911-7.

21. Lu SH, Wang JX, Niu YH, Yang J, Zhou J, Yuan YJ. Metabolic profiling reveals growth related FAME productivity and quality of Chlorella sorokiniana with different inoculum sizes. Biotechnol Bioeng. 2012;109(7):1651-62.

22. Yang S, Lu S-H, Yuan Y-J. Cerium elicitor-induced phosphatidic acid triggers apoptotic signaling development in Taxus cuspidata cell suspension cultures. Chem Phys Lipids. 2009;159(1):13-20.

23. Phukan MM, Chutia RS, Konwar B, Kataki R. Microalgae Chlorella as a potential bio-energy feedstock. Appl Energy. 2011;88(10):3307-12.

24. Tang H, Chen M, Garcia M, Abunasser N, Ng K, Salley SO. Culture of microalgae Chlorella minutissima for biodiesel feedstock production. Biotechnol Bioeng. 2011;108(10):2280-7.

25. Takenaka S, Kondo T, Nazeri S, Tamura Y, Tokunaga M, Tsuyama S, Miyatake K, Nakano Y. Accumulation of trehalose as a compatible solute under osmotic stress in Euglena gracilis Z. J Eukaryot Microbiol. 1997;44(6):609-13.
26. Wang J, Chen L, Tian X, Gao L, Niu X, Shi M, Zhang W. Global metabolomic and network analysis of Escherichia coli responses to exogenous biofuels. J Proteome Res. 2013;12(11):5302-12.

27. Laiakis EC, Morris GA, Fornace Jr AJ, Howie SR. Metabolomic analysis in severe childhood pneumonia in the Gambia, West Africa: findings from a pilot study. Plos One. 2010;5(9):e12655.

28. Regnault A, Chervin D, Chammai A, Piton F, Calvayrac R, Mazliak P. Lipid composition of Euglena gracilis in relation to carbon-nitrogen balance. Phytochemistry. 1995;40(3):725-33.

29. Barsanti L, Vismara R, Passarelli V, Gualtieri P. Paramylon ( $\beta-1,3-$ glucan) content in wild type and WZSL mutant of Euglena gracilis. Effects of growth conditions. J Appl Phycol. 2001;13(1):59-65.

30. Cordella C, Moussa I, Martel AC, Sbirrazzuoli N, Lizzani-Cuvelier L. Recent developments in food characterization and adulteration detection: Technique-oriented perspectives. J Agric Food Chem. 2002;50(7):1751-64.

31. Wang YX, Shi ML, Niu XF, Zhang XQ, Gao LJ, Chen L, Wang JX, Zhang WW. Metabolomic basis of laboratory evolution of butanol tolerance in photosynthetic Synechocystis sp PCC 6803. Microb Cell Fact. 2014;13:151.

32. Sui X, Niu X, Shi M, Pei G, Li J, Chen L, Wang J, Zhang W. Metabolomic analysis reveals mechanism of antioxidant butylated hydroxyanisole on lipid accumulation in Crypthecodinium cohnii. J Agric Food Chem. 2014;62(51): 12477-84.

33. Hunt RW, Chinnasamy S, Bhatnagar A, Das KC. Effect of Biochemical Stimulants on Biomass Productivity and Metabolite Content of the Microalga, Chlorella sorokiniana. Appl Biochem Biotechnol. 2010;162(8): 2400-14.

\section{Submit your next manuscript to BioMed Central and we will help you at every step:}

- We accept pre-submission inquiries

- Our selector tool helps you to find the most relevant journal

- We provide round the clock customer support

- Convenient online submission

- Thorough peer review

- Inclusion in PubMed and all major indexing services

- Maximum visibility for your research

Submit your manuscript at www.biomedcentral.com/submit
) Biomed Central 